\title{
Resveratrol modulates the Akt/GSK-3 $\beta$ signaling pathway in a middle cerebral artery occlusion animal model
}

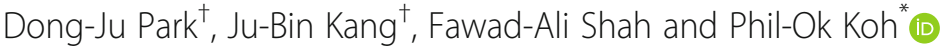

\begin{abstract}
Cerebral ischemia is a major cause of neurodegenerative disease. It induces neuronal vulnerability and susceptibility, and leads to neuronal cell death. Resveratrol is a polyphenolic compound that acts as an anti-oxidant. It exerts a neuroprotective effect against focal cerebral ischemic injury. Akt signaling pathway is accepted as a representative cell survival pathway, including proliferation, growth, and glycogen synthesis. This study investigated whether

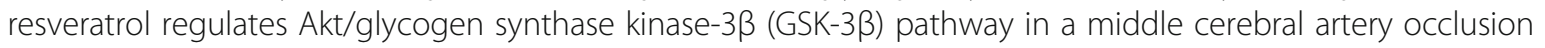
(MCAO)-induced ischemic brain injury. Adult male rats were intraperitoneally injected with vehicle or resveratrol (30 mg/kg) and cerebral cortices were isolated $24 \mathrm{~h}$ after MCAO. Neurological behavior test, corner test, brain edema measurment, and 2,3,5-triphenyltetrazolium chloride staining were performed to elucidate the neuroprotective effects of resveratrol. Phospho-Akt and phospho-GSK-3 $\beta$ expression levels were measured using Western blot analysis. MCAO injury led to severe neurobehavioral deficit, infraction, and histopathological changes in cerebral cortex. However, resveratrol treatment alleviated these changes caused by MCAO injury. Moreover, MCAO injury induced decreases in phospho-Akt and phospho-GSK-3 $\beta$ protein levels, whereas resveratrol attenuated these decreases. Phosphorylations of Akt and GSK-3 $\beta$ act as a critical role for the suppression of apoptotic cell death. Thus, our finding suggests that resveratrol attenuates neuronal cell death in MCAO-induced cerebral ischemia and Akt/GSK-3ß signaling pathway contributes to the neuroprotective effect of resveratrol.
\end{abstract}

Keywords: Akt, GSK-3ß, MCAO, Resveratrol

\section{Introduction}

Resveratrol is a polyphenolic compound that abundantly exists in grape and red wine. It is well known that resveratrol acts as an anti-oxidant and prevents oxidative damage in vascular and nervous system [1-3]. It also has a vasodilatory effect through stimulating nitric oxide production in endothelial cells [4]. Moreover, it exerts a neuroprotective effect against traumatic brain injury. Neuroprotective mechanisms of resveratrol are associated with its anti-inflammatory, anti-apoptotic, and antioxidant properties [5-7]. Resveratrol also plays a critical role in alleviation of neurodegenerative diseases such as

\footnotetext{
* Correspondence: pokoh@gnu.ac.kr

${ }^{\dagger}$ Dong-Ju Park and Ju-Bin Kang contributed equally to this work. Department of Anatomy, College of Veterinary Medicine, Research Institute of Life Science, Gyeongsang National University, 501 Jinju-daero, Jinju 52828, South Korea
}

Parkinson disease and Alzheimer's disease [8, 9]. Resveratrol protects neurons against focal cerebral ischemia and attenuates brain damage via activation of PI3K/Akt signaling pathway $[10,11]$.

Akt is a member of serine/threonine protein kinases family that performs an essential role in the modulation of cell development, proliferation, growth, and survival [12]. Akt is known as protein kinase B and extensively expressed in various tissues. Akt acts as a mediator of cell survival and regulates numerous downstream targets, such as glycogen synthase kinase-3 (GSK-3), forkhead transcription factors, and $\mathrm{Bad}$ [13-15]. It is also involved in multiple cellular processes, such as cell proliferation, transcription, apoptosis, and glucose metabolism $[15,16]$. Moreover, Akt has a critical function in neuronal survival during apoptotic injury [17]. Akt is substantially activated in the nervous system during cellular stress. Overexpression of Akt prevents apoptosis 
caused by growth factor depletion in cerebellar granule neurons [18].

GSK-3 is a serine/threonine protein kinases that regulates glycogen synthesis in response to insulin [19]. Moreover, it phosphorylates a broad range of substrates, including transcription factors, translation initiation factor, and eukaryotic initiation factor 2 [20, 21]. GSK-3 has two isoform, GSK- $3 \alpha$ and GSK-3 $\beta$. GSK-3 $\beta$ is a critical downstream target of the phosphoinositide 3-kinase (PI3K)/Akt signaling pathway and activity of GSK-3 $\beta$ is suppressed by Akt-mediated phosphorylation at serine 9 $[19,22]$. GSK-3 $\beta$ activates caspase-3 and leads to apoptotic cell death. Activated Akt inhibits GSK-3 $\beta$ function and mediates anti-apoptotic effect [23]. Previous studies reported that resveratrol improves neurological behavior score and attenuates brain damage on ischemic stroke $[24,25]$. Although many studies have been shown the neuroprotective effects of resveratrol, its mechanism has not been completely elucidated. Thus, this study confirmed the neuroprotective effects of resveratrol in middle cerebral artery occlusion (MCAO) animal model and investigated the underlying mechanisms of resveratrol through phosphorylations of Akt and its downstream target, GSK-3 $\beta$.

\section{Materials and methods}

\section{Experimental animals}

Adult male Sprague-Dawley rats $(220-230 \mathrm{~g}, n=32)$ were purchased from Samtako Co. (Animal Breeding Center, Osan Korea). All animals were housed in controlled light condition ( $12 \mathrm{~h}$ light/ $12 \mathrm{~h}$ dark cycle) and temperature $\left(25^{\circ} \mathrm{C}\right)$. Drinking water and feed were freely supplied. All experimental procedures were carried out according to the guideline of the Institutional Animal Care and Use Committee of Gyeongsang National University. Animals were divided into the following four groups: vehicle + sham, resveratrol + sham, vehicle + $\mathrm{MCAO}$, and resveratrol + MCAO (8 rats per group). Resveratrol $(30 \mathrm{mg} / \mathrm{kg}$, Sigma-Aldrich, St. Louis, MO, USA) was dissolved in $0.05 \%$ dimethyl sulfoxide (DMSO; Sigma-Aldrich) in phosphate buffered saline and immediately intraperitoneally injected [26]. Vehicle-treated animals were injected with only DMSO solution without resveratrol.

\section{Middle cerebral artery occlusion}

Rats were anesthetized with Zoletil $(50 \mathrm{mg} / \mathrm{kg}$; Virbac, Carros, France) and placed on a surgical plate with supine position. MCAO was surgically operated as previously described method [27]. A midline of neck skin was incised and right common carotid artery (CCA) was exposed. Right CCA was carefully separated from the adjacent muscles and nerves, and temporally blocked with a microvascular clip. Branches of external carotid artery
(ECA) were carefully dissected and ligated. Proximal end of the ECA was incised and a nylon filament 4/0 with flame-rounded tip was slowly inserted into the incision of ECA. Nylon filament was advanced into the lumen of internal carotid artery (ICA) until a slight resistance felt. It was inserted approximately 21 to $23 \mathrm{~mm}$ from the bifurcation of ECA and occluded the origin of middle cerebral artery. Filament was ligated with ECA and skin incision was sutured with black silk. Sham-operated animals were performed with the same surgical procedure without insertion of a nylon filament. Animals were kept on a heating pad to maintain body temperatures and given free access to feed and water. Neurological behavior test was performed $24 \mathrm{~h}$ after MCAO and expeditiously decapitated to reduce suffering. Whole brains were carefully removed and cerebral cortices were separated. Tissues were kept at $-70{ }^{\circ} \mathrm{C}$ or fixed in $4 \%$ neutral buffered paraformaldehyde (NBF) for further experimental procedure.

\section{Neurological behavior test and brain edema measurement}

Neurological behavior test was performed by previously described methods $[28,29]$. This test was scored with a five-point scoring system by following standard: no neurological deficit (0), normal posture but failed to extend forepaw on the contralateral side of ischemic region (1), normal posture and circling to the contralateral side of ischemic region (2), falling down to the contralateral side of ischemic region (3), no impulsive movement (4). Isolated right cerebral cortices were weighed for evaluation of brain edema. Measured weight was considered as wet weight. After weight measurement, right cerebral cortices were dried for $24 \mathrm{~h}$ at $100^{\circ} \mathrm{C}$ and weighed. This weight was taken as dry weight. The water content in the cerebral cortex was calculated as follows: [(wet weight - dry weight)/wet weight] $\times 100$.

\section{Corner test}

Corner test was performed to evaluate sensorimotor function by previously described manuals [30, 31]. Corner test apparatus was formed with two vertical boards $(30 \times 20 \times 1 \mathrm{~cm})$ that attached to each edge at the angle of $30^{\circ}$ with a small opening. The gap between two boards encouraged animals to move toward to the corner. When animals move to the corner, both side of vibrissae simultaneously touched boards. After vibrissae stimulation, animals rear against the corner and turn right or left side. Turning movement is recorded and rearing movement is excluded from measurement. Ten turns were counted for each trial and result was expressed as the number of right turn [30,31]. 


\section{Triphenyltetrazolium chloride staining}

Whole brains were carefully removed from skull and sliced into $2 \mathrm{~mm}$ coronary sections with a brain matrix (Ted Pella, Redding, CA, USA). Coronary section levels were marked using bregma level. Sliced tissues were reacted in $2 \%$ triphenyltetrazolium chloride (TTC; Sigma Aldrich) for $30 \mathrm{~min}$ at $37^{\circ} \mathrm{C}$ and fixed in $4 \%$ NBP for $24 \mathrm{~h}$. Images of stained tissues were scanned with Agfa ARCUS $1200^{\text {tm }}$ (Agfa-Gevaert, Mortsel, Belgium) and analyzed using Image-ProPlus 4.0 software (Media Cybernetics, Silver Spring, MD, USA) to evaluate the infarct volume. Ischemic area (\%) was calculated by the following formula: (infarction area/whole section area) $\times 100$.

\section{Hematoxylin and eosin staining}

Fixed brain tissues were washed with flowing tap water, dehydrated by series of graded ethyl alcohol from 70 to $100 \%$, and cleaned with xylene. Brain tissues were embedded in paraffin tank using embedding center (Leica, Westlar, Germany) and hardened as tissue blocks. Tissues were cut into $4 \mu \mathrm{m}$ sections and placed on glass slides in tissue bath (Leica). Sections were dried on slide warmer (Thermo Fisher Scientific, Waltham, MA, USA), deparaffinized with xylene, and rehydrated by series of graded ethyl alcohol from 100 to $70 \%$. They were stained with hematoxylin solution (Sigma-Aldrich) and eosin solution (Sigma-Aldrich), subsequently. Stained tissues were washed with tap water and dehydrated with series of graded ethyl alcohol. They were mounted with permount mounting solution (Thermo Fisher Scientific) and photographed using Olympus microscope (Olympus, Tokyo, Japan).

\section{Western blot analysis}

Right cerebral cortices were homogenized in lysis buffer [1\% Triton X-100, $1 \mathrm{mM}$ EDTA in PBS (pH 7.4)] containing $0.2 \mathrm{mM}$ phenylmethylsulfonyl fluoride. Homogenates were sonicated and centrifuged at $15,000 \mathrm{~g}$ for 30 min at $4{ }^{\circ} \mathrm{C}$. Supernatants were collected and protein concentrations were determined with a bicinchoninic acid protein assay kit (Pierce, Rockford, IL, USA) according to the manufacturer's manual. Protein samples were denatured by heating for $3 \mathrm{~min}$ at $100^{\circ} \mathrm{C}$ and cooled for $1 \mathrm{~min}$ in ice. Equal amount of proteins $(30 \mu \mathrm{g})$ were loaded and electrophoresed in $10 \%$ sodium dodecyl sulfate polyacrylamide gel electrophoresis gel. Separated proteins were transferred to polyvinylidene fluoride membranes. Membranes were incubated in 5\% skim milk with Tris-buffered saline containing $0.1 \%$ Tween-20 (TBST) for $1 \mathrm{~h}$. After washing with TBST, membranes were incubated for overnight at $4{ }^{\circ} \mathrm{C}$ with following primary antibodies: anti-Akt, anti-phosphoAkt (Serine 473), anti-GSK-3 $\beta$, anti-phospho-GSK-3 $\beta$ (Serine 9), (diluted 1:1000, Cell Signaling Technology,
Beverly, MA, USA), and anti- $\beta$-actin (diluted 1:1000, Millipore, Billerica, MA, USA). Membranes were washed three times with TBST for $10 \mathrm{~min}$ and treated with their respective secondary antibody (horseradish peroxidaseconjugated anti-rabbit IgG or anti-mouse IgG, diluted 1: 5000, Cell Signaling Technology) for $2 \mathrm{~h}$ at room temperature. After washing with TBST, membranes were reacted with enhanced chemiluminescence Western Blotting detection reagents (GE Healthcare, Chicago, IL, USA) for the detection of immunoreactive protein bands. Membranes were exposed on Fuji medical X-ray film (Fuji Film, Tokyo, Japan) to visualize immunoreactive bands. The intensity value of protein bands were analyzed with Image J software (National Institutes of Health, Bethesda, MD, USA).

\section{Statistical analysis}

All experiment data were presented as the mean \pm standard error of means (S.E.M.). The results of each group were compared by two-way analysis of variance (ANOVA) followed by post-hoc Scheffe's test. $P<0.05$ was regarded as statistically significant.

\section{Results}

We confirmed that MCAO-induced cerebral ischemia leads to neurological behavior dysfunction and brain infarction. As a further study, we showed that resveratrol prevented MCAO-induced these changes. We evaluated neurological damage by neurological behavior deficit scoring and brain edema measurement. MCAOoperated animals with vehicle showed severe neurological symptoms, such as involuntary circling and seizure. Resveratrol treatment in MCAO-operated animals attenuated these symptoms and showed only mild neurological symptoms. Resveratrol significantly reduced MCAO-induced increase in neurological deficit scores. Neurological deficit scores were $3.25 \pm 0.24$ and $1.87 \pm$ 0.38 in vehicle+ MCAO and resveratrol + MCAO animals, respectively (Fig. 1a). Results of corner test showed the direction bias of the response by bilateral stimuli. The number of right turn indicates the ipsilateral side of insulted brain hemisphere. Sham-operated animals appeared as a similar pattern in turning left and right direction. However, MCAO animals with vehicle showed rightward-preferred turning pattern, resveratrol treatment reduced the number of rightward-preferred turning. Numbers of right turn were $9.45 \pm 0.35$ and $7.1 \pm$ 0.53 in vehicle+ MCAO and resveratrol + MCAO animals, respectively (Fig. 1b). Water contents of cerebral cortices were measured to assess the degree of brain edema. MCAO-operated animals with vehicle showed severe brain edema, resveratrol treatment alleviated MCAO-induced excessive brain edema. Water contents 

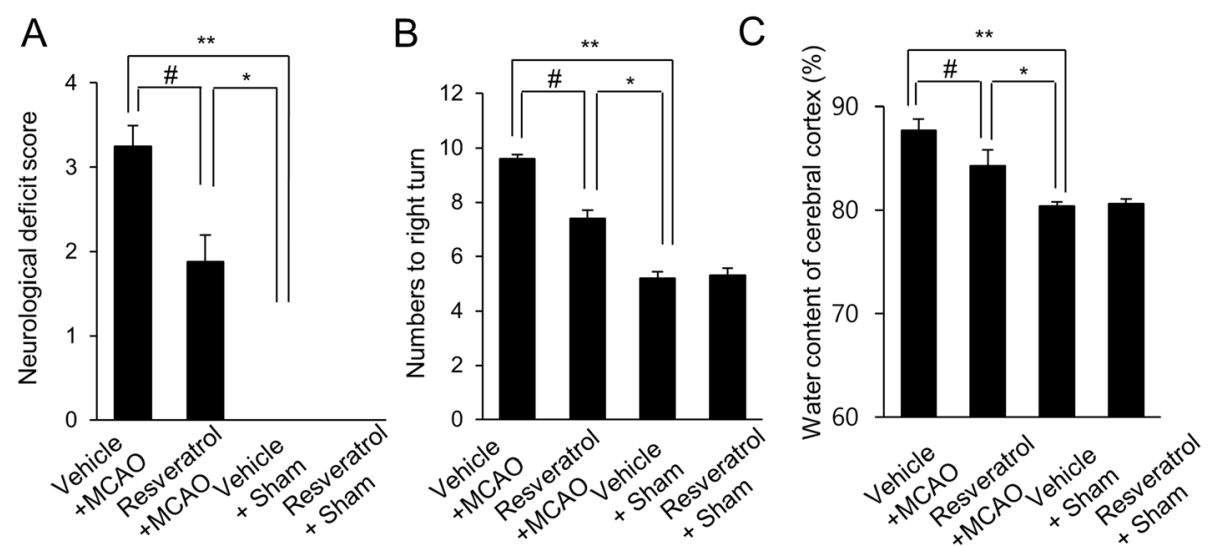

Fig. 1 Neurobehavioral scores (a), corner test (b), and brain edema measurement (c) in vehicle + sham, resveratrol + sham, vehicle + middle cerebral artery occlusion (MCAO), and resveratrol + MCAO animals. Resveratrol attenuated the neurological behavior deficits and brain edema induced by ischemic stroke. Data $(n=8)$ are represented as the mean \pm S.E.M. ${ }^{*} p<0.01,{ }^{* *} p<0.05$ vs. vehicle + sham animals, $\# p<0.05$ vs. vehicle + MCAO animals

were $87.73 \pm 1.12$ and $84.32 \pm 1.51 \%$ in vehicle + MCAO and resveratrol + MCAO animals, respectively (Fig. 1c).

TTC staining was performed to evaluate the infarct volume. Infarct area was stained distinct red color, while infarct area remained as unstained white color. Results of TTC staining showed a widespread infarction in vehicle + MCAO animals. However, infarct area was significantly reduced in resveratrol + MCAO animals. Moreover, infarct area was not observed in shamoperated animals regardless of vehicle or resveratrol treatment (Fig. 2a). Volumes of infarct area were $26.31 \pm 2.31 \%$ and $17.08 \pm 2.70 \%$ in vehicle $+\mathrm{MCAO}$ and resveratrol + MCAO animals, respectively (Fig. 2b). Morphological study showed the histopathological changes in cerebral cortex of MCAO-operated animals (Fig. 2c-f). We observed shape of typical pyramidal cells with large and round nucleus in sham-operated animals. They also had intact cytoplasm and dendrites. However, we found shrunken neurons with abnormal morphology in MCAO-operated animals with vehicle. They had condensed and shrunken nuclei and numerous vacuoles in cytoplasm. Resveratrol treatment attenuated MCAOinduced these pathological changes. Pyknotic nuclei and vacuoles in cytoplasm were reduced in MCAO animals with resveratrol.

Western blot analysis result showed the expression changes of phospho-Akt and phospho-GSK-3 $\beta$ in cerebral cortex between vehicle + MCAO and resveratrol + MCAO animals (Fig. 3). phospho-Akt and phosphoGSK-3 $\beta$ protein expression levels were significantly decreased in cerebral cortex of vehicle + MCAO animals. However, these decreases were recovered in resveratrol + MCAO animals (Fig. 3a). Phospho-Akt levels were $0.06 \pm 0.02$ in vehicle $+\mathrm{MCAO}$ and $0.74 \pm 0.08$ in resveratrol + MCAO animals. Phospho-GSK-3 $\beta$ levels were
$0.30 \pm 0.02$ and $0.61 \pm 0.06$ in vehicle + MCAO and resveratrol $+\mathrm{MCAO}$ animals, respectively (Fig. $3 \mathrm{~b}$ ).

\section{Discussion}

Cerebral ischemia causes various neurological dysfunctions, including complex neuromuscular dysfunction and neurological behavior deficit [32]. It leads to the formation of pathological lesions, such as swollen axon, neuronal perikaryal damage, and brain edema [33, 34]. Moreover, it is accepted that free radical causes neuronal damage in cerebral ischemia. Resveratrol is a strong anti-oxidant that abundantly presents in grape seed. It suppresses infarct size and improves neurological functions in ischemic brain injury [24, 35-37]. In our study, we indirectly treated resveratrol through intraperitoneal injection. It is reported that resveratrol can distribute in brain through intraperitoneal injection [38]. Resveratrol also has a neuroprotective effect through intraperitoneal injection against kainic acid-induced brain damage, global cerebral ischemia and focal ischemia [38, 39]. Also, resveratrol exerts neuroprotective effect against cerebral ischemic injury by modulating mitochondrial dysfunction [37]. Resveratrol protects neurons against cerebral ischemia by inhibiting inflammation and apoptosis [40]. Moreover, it also prevents brain damage by reducing oxidative stress and ameliorating mitochondria damage in a cerebral ischemia [41]. We confirmed the neuroprotective effect of resveratrol in MCAO animal model. Resveratrol alleviated MCAO-induced neurological behavior deficits. Moreover, our previous study has been shown that resveratrol regulates the expression of various proteins that associated with oxidative stress in focal cerebral ischemia [26].

It is accepted that resveratrol exerts a neuroprotective effect by modulating of various molecular mechanism. 
A

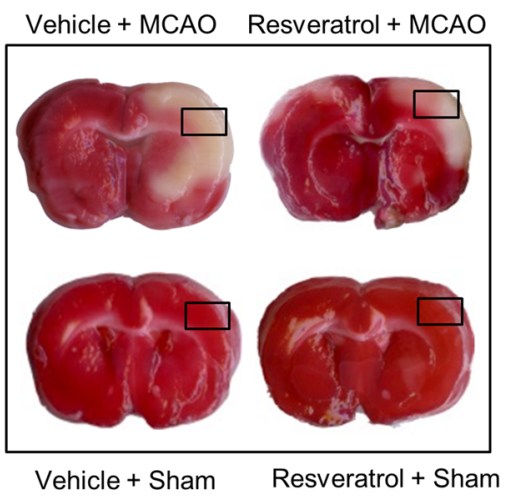

B

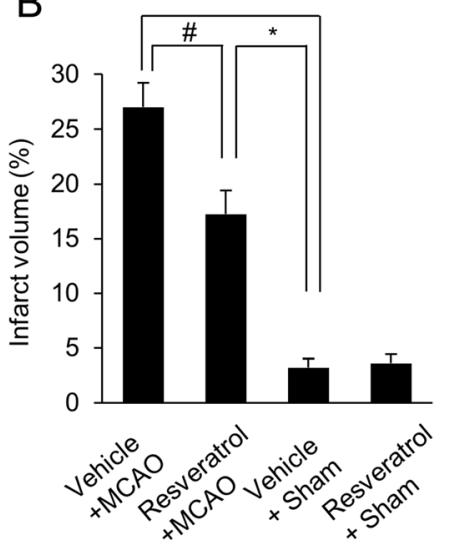

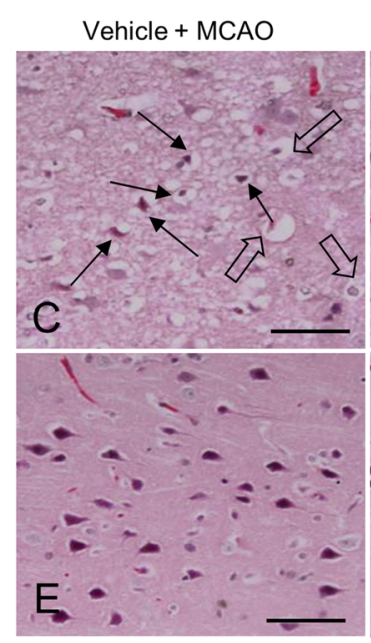

Vehicle + Sham

Resveratrol + MCAO

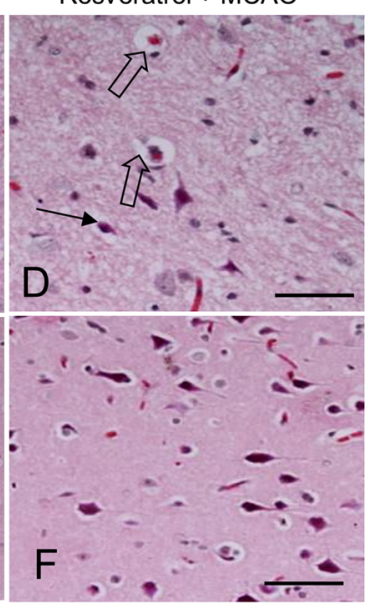

Resveratrol + Sham

Fig. 2 Representative photograph of TTC staining (a), infarct volume (b), and hematoxylin and eosin staining (c-f) in cerebral cortex of vehicle + sham, resveratrol + sham, vehicle + middle cerebral artery occlusion (MCAO), and resveratrol + MCAO animals. Infarct volume was calculated by ratio of infarction area to total area. Resveratrol attenuated the MCAO-induced infarct region. C-F photos indicate the square areas of A. Arrows indicate shrunken and condensed nuclei and open arrows indicate swelled and vacuolated forms. Scale bar $=100 \mu \mathrm{m}$. Data $(n=4)$ are represented as the mean \pm S.E.M. ${ }^{*} p<0.01,{ }^{* *} p<0.05$ vs. vehicle + sham animals, $\# p<0.05$ vs. vehicle + MCAO animals

Resveratrol decreases the elevated level of matrix metalloproteinase 9 caused by cerebral ischemia [42]. Moreover, resveratrol prevented the brain from ischemia through modulation of $\mathrm{Ca}^{2+}$ response element-binding protein [43]. Resveratrol protects hippocampal neurons against ischemic injury-induced damage via the extracellular signal regulated kinase signaling pathway [44]. It also exerts its neuroprotective effect by modulating of PI3K/Akt signaling pathway [11]. Resveratrol also attenuates ischemic brain damage through upregulation of inflammatory factors, interleukin-1 beta (IL-1 $\beta$ ) and tumor necrosis factor-alpha (TNF- $\alpha)$ [11]. PI3K/Akt signaling pathway plays a key role for cell growth and cell survival. Akt also exerts anti-apoptotic effect by inhibiting the pro-apoptotic proteins, such as Bad and forkhead transcription factors [14]. Akt prevents injury-induced neuronal death and accelerates axonal regeneration [45]. It also contributes to neuronal migration and survival
[46-49]. Our previous studies demonstrated that MCAO decreases Akt phosphorylation and consecutively reduces phosphorylation of its down-stream targets such as Bad, forkhead transcription factors, and GSK-3 beta [50-53]. Moreover, neuroprotective agents alleviate MCAO-induced decreases in phosphorylation of Akt and its down-stream targets [50-53]. MCAO clearly results in decreased levels of phospho-Akt and phospho-GSK-3 $\beta$ and neuroprotective agents attenuate the decreases in the levels of these proteins $[50,53]$. The present study showed that focal cerebral ischemia significantly reduces phosphoAkt and phospho-GSK-3 $\beta$ expressions in cerebral cortex. Moreover, resveratrol delays the progression of 6hydroxydopamine-induced motor dysfunction and apoptosis by activating PI3K/Akt cell survival pathway [54]. Phosphorylation of Akt is upregulated in early stage of ischemic stroke as a defensive reaction of neuronal cells whether this upregulation is terminated in late stage of 

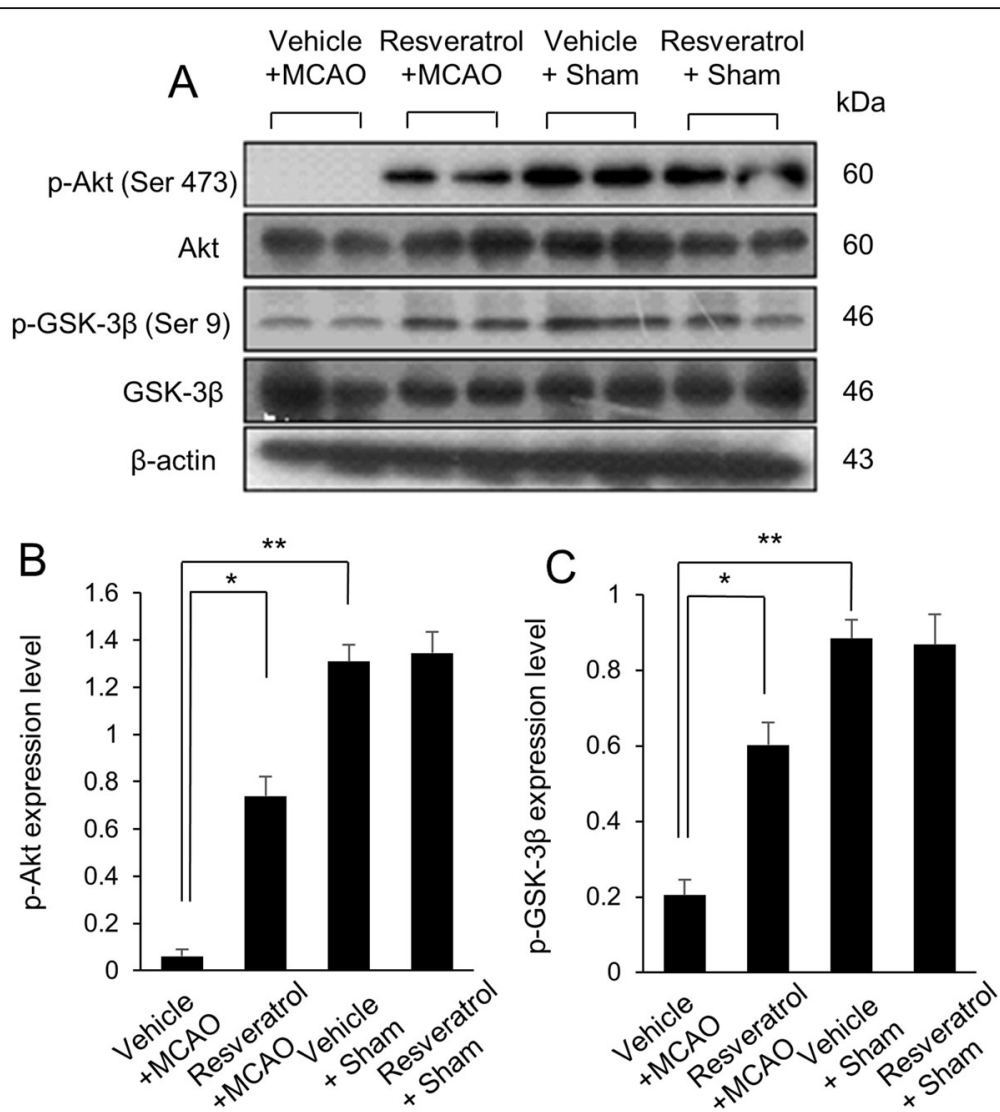

Fig. 3 Western blot analysis of phospho-Akt and phospho-GSK-3 $\beta$ in cerebral cortex of vehicle + sham, resveratrol + sham, vehicle + middle cerebral artery occlusion (MCAO), and resveratrol + MCAO animals (a). Densitometric analysis is represented as a ratio of phospho-Akt (b) and phospho-GSK-3B (c) intensity to $\beta$-actin intensity. Data $(n=4)$ are shown as the mean \pm S.E.M. ${ }^{*} p<0.01,{ }^{* *} p<0.05$ vs. vehicle + sham animals, \# $p<0.05$ vs. vehicle + MCAO animals

ischemic stroke [55]. In our study, we performed MCAO for $24 \mathrm{~h}$ which is a late stage of ischemic stroke and phosphorylation of Akt is suppressed. However, resveratrol attenuated MCAO-induced decrease in phospho-Akt. Decrease of phospho-Akt induces apoptotic cell death and consequently inhibits cell survival. In addition, we elucidated the changes of GSK-3 $\beta$ by resveratrol treatment in MCAO-induced cerebral ischemia. Our results showed that resveratrol treatment in cerebral ischemia regulates Akt and its downstream target, GSK-3 $\beta$. Focal cerebral ischemia caused by MCAO decreases phospho-GSK-3 $\beta$ levels and resveratrol alleviates decreases in phosphoGSK-3 $\beta$. GSK-3 $\beta$ induces cell death by increasing caspase3 activity in ischemic injury [56]. Thus, it is considered that phosphorylation of GSK-3 $\beta$ by Akt is a critical process for the inhibition of its pro-apoptotic activity. Previous study showed that resveratrol significantly downregulates cleaved caspase- 3 and bax expressions, upregulates bcl-2 expression in stroke condition [57]. Moreover, resveratrol alleviates nerve injury in cerebral ischemia via upregulation of hippocampal $\mathrm{Bcl}-2$ [58]. Inactivation of caspase-3 leads to the suppression of apoptotic cell death. Resveratrol improves neurological function and neuronal damage, alleviates neuronal apoptosis in cerebral ischemia. We clearly demonstrated that resveratrol prevents the ischemic injury-induced decrease in phospho-GSK-3 $\beta$ expression. The maintenance of phospho-GSK-3 $\beta$ by resveratrol in cerebral ischemia mediates the inactivation of caspase- 3 and suppression of apoptosis $[56,57]$. The Akt/ GSK-3 $\beta$ signaling pathway is an important mechanism of neuroprotective effect. We elucidated the fact that resveratrol prevents the MCAO injury-induced reductions in phospho-Akt and phospho-GSK-3 $\beta$.

\section{Conclusions}

In this study, we confirmed that resveratrol improves neuronal damage against MCAO-induced cerebral ischemic injury. Furthermore, our study reveals that resveratrol has a neuroprotective effects by regulating the Akt/ GSK-3 $\beta$ signaling pathway. Therefore, this study can suggest the underlying mechanisms in neuroprotective effect of resveratrol attributing to neuronal cell survival. 


\section{Abbreviations}

CCA: Common carotid artery; DMSO: Dimethyl sulfoxide; ECA: External carotid artery; GSK-3: Glycogen synthase kinase-3; GSK-3 $\beta$ : Glycogen synthase

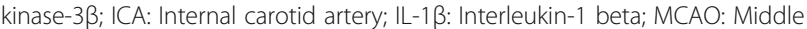
cerebral artery occlusion; NBF: Neutral buffered paraformaldehyde; NRF: National research foundation of korea; PI3K: Phosphoinositide 3-kinase; TBST: Tris-buffered saline containing 0.1\% Tween-20; TNF-a: Tumor necrosis factor-alpha; TTC: Triphenyltetrazolium chloride

\section{Acknowledgements}

Not applicable.

\section{Authors' contributions}

D-JP, J-BK and F-AS performed experiment, organized and analyzed data. POK designed the experiment, organized data, wrote and correct the manuscript, and managed general research and drafting. All authors read and approved this final manuscript.

\section{Funding}

This research was supported by the National Research Foundation of Korea (NRF) grant funded by the Korea government (MEST) (NRF2018R1D1A1B07044074)

\section{Availability of data and materials}

The data that support the findings of this study are available on request from the corresponding author on reasonable request.

\section{Competing interests}

The authors declare that they have no competing interests.

Received: 2 August 2019 Accepted: 25 September 2019

Published online: 15 October 2019

\section{References}

1. Fremont L. Biological effects of resveratrol. Life Sci. 2000;66(8):663-73.

2. Chanvitayapongs S, Draczynska-Lusiac B, Sun AY. Amelioration of oxidative stress by antioxidants and reveratrol in PC12 cells. Neuroreport. 1997;8(6): 1499-502

3. Raval A, Lin H, Dave K, DeFazio A, Della Morte D, Kim E, Perez-Pinzon M. Resveratrol and ischemic preconditioning in the brain. Curr Med Chem. 2008;15(15):1545

4. Chen CK, Pace-Asciak CR. Vasorelaxing activity of resveratrol and quercetin in isolate rat aorta. Gen Pharmacol. 1996;27(2):363-6.

5. de Queiroz KB, Dos Santos Fontes Pereira T, Araújo MSS, Gomez RS, Coimbra RS. Resveratrol acts anti-inflammatory and neuroprotective in an infant rat model of pneumococcal meningitis by modulating the hippocampal miRNome. Mol Neurobiol. 2008;55(12):8869-84.

6. Meng Z, Li J, Zhao H, Liu H, Zhang G, Wang L, Hu HE, Li DI, Liu M, Bi F, Wang X, Tian G, Liu Q, Buren B. Resveratrol relieves ischemia-induced oxidative stress in the hippocampus by activating SIRT1. Exp Ther Med. 2015:10(2):525-30

7. Shin JY, Seo MA, Choi EJ, Kim JK, Seo ES, Lee JH, Chung HL, Kim WT Neuroprotective effects of resveratrol via anti-apoptosis on hypoxicischemic brain injury in neonatal rats. Korean J Pediatr. 2008;51(10):1102-11.

8. Rege SD, Geetha T, Griffin GD, Broderick TL, Babu JR. Neuroprotective effects of resveratrol in Alzheimer disease pathology. Front Aging Neurosci. 2014:6:218.

9. Xia D, Sui R, Zhang Z. Administration of resveratrol improved Parkinson's disease-like phenotype by suppressing apoptosis of neurons via modulating the MALAT1/miR-129/SNCA signaling pathway. J Cell Biochem. 2019;120(4):4942-51.

10. Dou Z, Rong X, Zhao E, Zhang L, LV Y. Neuroprotection of resveratrol against focal cerebral ischemia/reperfusion injury in mice through a mechanism targeting gut-brain axis. Cell Mol Neurobiol. 2019;39(6):883-98

11. Lei J, Chen Q. Resveratrol attenuates brain damage in permanent focal cerebral ischemia via activation of PI3K/Akt signaling pathway in rats. Neurol Res. 2018;40(12):1014-20.

12. Testa JR, Bellacosa A. AKT plays a central role in tumorigenesis. Proc Nat Acad Sci U S A. 2001;98(20):10983-5.
13. Datta SR, Dudek H, Tao X, Masters S, Fu H, Gotoh Y, Greenberg ME. Akt phosphorylation of BAD couples survival signals to the cell-intrinsic death machinery. Cell. 1997;91(2):231-41.

14. Rokutanda S, Fujita T, Kanatani N, Yoshida CA, Komori H, Liu W, Mizuno A, Komori T. Akt regulates skeletal development through GSK3, mTOR, and FoxOs. Dev Biol. 2009;328(1):78-93.

15. Song G, Ouyang G, Bao S. The activation of Akt/PKB signaling pathway and cell survival. J Cell Mol Med. 2005:9(1):59-71.

16. Chin YR, Toker A. Function of Akt/PKB signaling to cell motility, invasion and the tumor stroma in cancer. Cell Signal. 2009;21(4):470-6.

17. Morrison RS, Kinoshita Y, Johnson MD, Ghatan S, Ho JT, Garden G. Neuronal survival and cell death signaling pathways. Adv Exp Med Biol. 2002;513:41-86.

18. Dudek H, Datta SR, Franke TF, Birnbaum MJ, Yao R, Cooper GM, Segal RA, Kaplan DR, Greenberg ME. Regulation of neuronal survival by the serinethreonine protein kinase Akt. Science. 1997;275(5300):661-5.

19. Cross DAE, Alessi DR, Cohen P, Andjelkovich M, Hemmings BA. Inhibition of glycogen synthase kinase-3 by insulin mediated by protein kinase B. Nature. 1995;378(6559):785-9.

20. Pap M, Cooper GM. Role of glycogen synthase kinase-3 in the phosphatidylinositol 3-kinase/Akt cell survival pathway. J Biol Chem. 1998; 273(32):19929-32.

21. Welsh GI, Miller CM, Loughlin AJ, Price NT, Proud CG. Regulation of eukaryotic initiation factor elF2B: glycogen synthase kinase-3 phosphorylates a conserved serine which undergoes dephosphorylation in response to insulin. FEBS Lett. 1998:421(2):125-30.

22. Srivastava AK, Pandey SK. Potential mechanism(s) involved in the regulation of glycogen synthesis by insulin. Mol Cell Biochem. 1998;182(1-2):135-41.

23. Romorini L, Garate X, Neiman G, Luzzani C, Furmento VA, Guberman AS, Sevlever GE, Scassa ME, Miriuka SG. AKT/GSK3 $\beta$ signaling pathway is critically involved in human pluripotent stem cell survival. Sci Rep. 2016:6:35660

24. Dong W, Li N, Gao D, Zhen H, Zhang X, Li F. Resveratrol attenuates ischemic brain damage in the delayed phase after stroke and induces messenger RNA and protein express for angiogenic factors. J Vasc Surg. 2008:48(3):709-14.

25. Shin JA, Lee H, Lim YK, Koh Y, Choi JH, Park EM. Therapeutic effects of resveratrol during acute periods following experimental ischemic stroke. J Neuroimmunol. 2010;227(1-2):93-100.

26. Shah FA, Gim SA, Kim MO, Koh PO. Proteomic identification of proteins differentially expressed in response to resveratrol treatment in middle cerebral artery occlusion stroke model. J Vet Med Sci. 2014;76(10):1367-74.

27. Longa EZ, Weinstein PR, Carlson S, Cummins R. Reversible middle cerebral artery occlusion without craniectomy in rats. Stroke. 1989;20(1):84-91.

28. Jin Z, Liang J, Wang J, Kolattukudy PE. MCP-induced protein 1 mediates the minocycline-induced neuroprotectionagainst cerebral ischemia/reperfusion injury in vitro and in vivo. J Neuroinflammation. 2015;12:39.

29. Shamsaei N, Erfani S, Fereidoni M, Shahbazi A. Neuroprotective effects of exercise on brain edema and neurological movement disorders following the cerebral ischemia and reperfusion in rats. Basic Clin Neurosci. 2017:8(1):77-84

30. Schallert T, Upchurch M, Lobaugh N, Farrar SB, Spirduso WW, Gilliam P, Vaughn D, Wilcox RE. Tactile extinction: distinguishing between sensorimotor and motor asymmetries in rats with unilateral nigrostriata damage. Pharmacol Biochem Behav. 1982:16(3):455-62.

31. Zhang L, Schallert T, Zhang ZG, Jiang Q, Arniego P, Li Q, Lu M, Chopp M. A test for detecting long-term sensorimotor dysfunction in the mouse after focal cerebral ischemia. J Neurosci Methods. 2002;117(2):207-14.

32. Petullo D, Masonic K, Lincoln C, Wibberley L, Teliska M, Yao DL. Model development and behavioral assessment of focal cerebral ischemia in rats. Life Sci. 1999;64(13):1099-108.

33. Valeriani V, Dewar D, McCulloch J. Quantitative assessment of ischemic pathology in axons, oligodendrocytes, and neurons: attenuation of damage after transient ischemia. J Cereb Blood Flow Metab. 2000;20(5):765-71.

34. Hatashita S, Hoff JT, Salamat SM. Ischemic brain edema and the osmotic gradient between blood and brain. J Cereb Blood Flow Metab. 1988;8(4):552-9.

35. Huang SS, Tsai MC, Chih CL, Hung LM, Tsai SK. Resveratrol reduction of infarct size in long-Evans rats subjected to focal cerebral ischemia. Life Sci. 2001:69(9):1057-65.

36. Lin JF, Lin SM, Chih CL, Nien MW, Su HH, Hu BR, Huang SS, Tsai SK. Resveratrol reduces infarct size and improves ventricular function after myocardial ischemia in rats. Life Sci. 2008;83(9-10):313-7. 
37. Yousuf S, Atif F, Ahmad M, Hoda N, Ishrat T, Khan B, Islam F. Resveratrol exerts its neuroprotective effect by modulating mitochondrial dysfunctions and associated cell death during cerebral ischemia. Brain Res. 2009;1250:242-53.

38. Wang $Q, X u$ J, Rottinghaus GE, Simonyi A, Lubahn D, Sun GY, Sun AY. Resveratrol protects against global cerebral ischemic injury in gerbils. Brain Res. 2002;958(2):439-47.

39. Virgili M, Contestabile A. Partial neuroprotection of in vivo excitotoxic brain damage by chronic administration of the red wine antioxidant agent, transresveratrol in rats. Neurosci Lett. 2000;281 (2-3):123-6.

40. Fang L, Gao H, Zhang W, Zhang W, Wang Y. Resveratrol alleviates nerve injury after cerebral ischemia and reperfusion in mice by inhibiting inflammation and apoptosis. Int J Clin Exp Med. 2015;8(3):3219-26.

41. Wang R, Liu YY, Liu XY, Jia SW, Zhao J, Cui D, Wang L. Resveratrol protects neurons and the myocardium by reducing oxidative stress and ameliorating mitochondria damage in a cerebral ischemia rat model. Cell Physiol Biochem. 2014;34(3):854-64.

42. Gao D, Zhang X, Jiang X, Peng Y, Huang W, Cheng G, Song L. Resveratrol reduces the elevated level of MMP-9 induced by cerebral ischemiareperfusion in mic. Life Sci. 2006;78(22):2564-70.

43. Lin Y, Chen F, Zhang J, Wang T, Wei X, Wu J, Feng Y, Dai Z, Wu Q Neuroprotective effect of resveratrol on ischemia/reperfusion injury in rats through TRPC6/CREB pathways. J Mol Neurosci. 2013;50(3):504-13.

44. Li Z, Fang F, Wang Y, Wang L. Resveratrol protects CA1 neurons against focal cerebral ischemic reperfusion-induced damage via the ERK-CREB signaling pathway in rats. Pharmacol Biochem Behav. 2016;146-147:21-7.

45. Namikawa K, Honma M, Abe K, Takeda M, Mansur K, Obata T, Miwa A, Okado H, Kiyama H. Akt/protein kinase B prevents injury-induced motoneuron death and accelerates axonal regeneration. J Neurosci. 2000; 20(8):2875-86.

46. Beffert U, Morfini G, Bock HH, Reyna H, Brady ST, Herz J. Reelin-mediated signaling locally regulates protein kinase B/Akt and glycogen synthase kinase 3beta. J Biol Chem. 2002;277(51):49958-64.

47. Franke TF. PI3K/Akt:getting it right matters. Oncogene. 2008;27(50):6473-88.

48. Niizuma K, Endo H, Chan PH. Oxidative stress and mitochondrial dysfunction as determinants of ischemic neuronal death and survival. J Neurochem. 2009;109(1):133-8.

49. Freyberg Z, Ferrando SJ, Javitch JA. Roles of the Akt/GSK-3 and Wnt signaling pathway sinschizophrenia and antipsychotic drug action. Am J Psychiatry. 2010;167(4):388-96.

50. Gim SA, Sung JH, Shah FA, Kim MO, Koh PO. Ferulic acid regulates the AKT/ GSK-3B/CRMP-2 signaling pathway in a middle cerebral artery occlusion animal model. Lab Anim Res. 2013;29(2):63-9.

51. Koh PO. Ferulic acid prevents the cerebral ischemic injury-induced decrease of Akt and bad phosphorylation. Neurosci Lett. 2012;507(2):156-60.

52. Koh PO. Melatonin prevents the injury-induced decline of Akt/forkhead transcription factors phosphorylation. J Pineal Res. 2008:45(2):199-203.

53. Koh PO, Cho GJ, Choi WS. 17beta-estradiol pretreatment prevents the global ischemic injury-induced decrease of Akt activation and bad phosphorylation in gerbils. J Vet Med Sci. 2006;68(10):1019-22.

54. Huang N, Zhang Y, Chen M, Jin H, Nie J, Luo Y, Zhou S, Shi J, Jin F. Resveratrol delays 6-hydroxydopamine-induced apoptosis by activating the PI3K/Akt signaling pathway. Exp Gerontol. 2019;124:110653.

55. Shibata M, Yamawaki T, Sasaki T, Hattori H, Hamada J, Fukuuchi Y, Okano H, Miura M. Upregulation of Akt phosphorylation at the early stage of middle cerebral artery occlusion in mice. Brain Res. 2002;942(1-2):1-10.

56. Brywe KG, Mallard C, Gustavsson M, Hedtjärn M, Leverin AL, Wang X, Blomgren K, Isgaard J, Hagberg H. IGF-I neuroprotection in the immature brain after hypoxia-ischemia, involvement of Akt and GSK3beta? Eur J Neurosci. 2005;21(6):1489-502.

57. Hou Y, Wang K, Wan W, Cheng Y, Pu X, Ye X. Resveratrol provides neuroprotection by regulating the JAK2/STAT3/PI3K/AKT/mTOR pathway after stroke in rats. Genes Dis. 2018;5(3):245-55.

58. Li Z, Pang L, Fang F, Zhang G, Zhang J, Xie M, Wang L. Resveratrol attenuates brain damage in a rat model of focal cerebral ischemia via upregulation of hippocampal Bcl-2. Brain Res. 2012;23(1450):116-24.

\section{Publisher's Note}

Springer Nature remains neutral with regard to jurisdictional claims in published maps and institutional affiliations.

\section{Ready to submit your research? Choose BMC and benefit from:}

- fast, convenient online submission

- thorough peer review by experienced researchers in your field

- rapid publication on acceptance

- support for research data, including large and complex data types

- gold Open Access which fosters wider collaboration and increased citations

- maximum visibility for your research: over $100 \mathrm{M}$ website views per year

At BMC, research is always in progress.

Learn more biomedcentral.com/submissions 